\title{
Steady plastic wave fronts and scale universality of strain localization in metals and ceramics
}

\author{
Yuriy Bayandin, Natalia Saveleva, Oleg Naimark \\ Institute of Continuous Media Mechanics Ural Branch Russian Academy of Sciences, 1, Ac. Korolev str., Perm 614013, Russia \\ buv@icmm.ru, bttps://orcid.org/0000-0002-1824-1940 \\ saveleva@icmm.ru,bttps://orcid.org/0000-0003-3756-7695 \\ naimark@icmm.ru, bttps://orcid.org/0000-0001-6537-1177
}

\begin{abstract}
Mechanisms of structural relaxation are linked with the metastability of nonequilibrium potential of solid with defects and the generation of collective modes of defects responsible for the plastic strain and damage localization. It is shown that spatial-temporal dynamics of collective modes (auto-solitary and blow-up dissipative structures) provide the anomalous relaxation ability of nonlinear system "solid with defects" in the conditions of the specific type of criticality - structural-scaling transition. These modes have the nature of self-similar solutions of evolution equations for damage parameter (defect-induced strain) and represent the "universality class" providing the four power law for a steady plastic front, splitting of an elastoplastic shock wave front, and elastic precursor decay kinetics. Widerange constitutive equations reflecting the linkage between defect-induced mechanisms and structural relaxation are used in the numerical simulation for shock wave loading of metals and ceramics in the comparison with experiments.
\end{abstract}

KEYwORDS. Metals, Ceramics; Shock waves; Self-similarity; Defects; Spall strength.

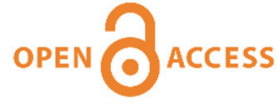

Citation: Bayandin, Yu., Saveleva, N., Naimark, O., Steady plastic wave fronts and scale universality of strain localization in metals and ceramics, Frattura ed Integrità Strutturale, 49 (2019) 243-256.

Received: 31.03 .2019

Accepted: 29.05.2019 Published: 01.07.2019

Copyright: (C) 2019 This is an open access article under the terms of the CC-BY 4.0, which permits unrestricted use, distribution, and reproduction in any medium, provided the original author and source are credited.

\section{INTRODUCTION}

$\mathrm{P}$ hysical mechanisms leading to the fracture of solids are associated with multiscale structural relaxation phenomena caused by mesoscopic defects. The conditions of dynamic high-speed loading of solid with characteristic times close to the relaxation times of structural stresses provide a unique opportunity to study experimentally the kinetics of the processes of failure and deformation in the strain rate range of $10^{3}-10^{8} \mathrm{~s}^{-1}$.

The deformation and failure of solids under shock wave loading are accompanied by pronounced self-similar regularities. In the experimental investigation of Barker [1], Swegle and Grady [2,3] the self-similarity of plastic wave fronts in metals and non-metals at strain rates of $\sim 10^{5} \mathrm{~s}^{-1}$ was established. Modern statements about the mechanisms of deformation and fracture, the experimental possibilities of using the technique of shock wave experiment and high time resolution 
recording systems can be the basis for the development of universal methods for investigating the rheological properties and the destruction of condensed matter due to multiscale structural effects. Experimental data on structured plane shock front are presented for a wide class of materials - both metals and nonmetallic materials [2, 3].

The description of mechanical properties of solid over a range of strain rates about $10^{3}-10^{8} \mathrm{~s}^{-1}$ due to multiscale mechanisms of structural relaxation mechanisms was proposed in the form of the corresponding constitutive equations of elastoviscoplastic material, as well as the kinetic equations of damage accumulation. This approach is combined with advanced experimental methods that allow the "in-situ" study of relaxation properties and damage accumulation stages (up to failure) using the Doppler interferometry. The measurements are realized on time scales close to the times of structural relaxation and reflecting the multilevel kinetics of nucleation and growth of defects, the kinetics of phase transformations in shocked materials. The peculiarity of deformation behavior and the development of failure under intense loadings is the proximity of the times of structural relaxation caused by the kinetics of defects with relaxation times that determine the development of plastic deformation and damage in the material. These features and the nonlinear kinetics of defects play a major role and are linked with key problems of describing the self-similar structure of wave fronts, the relaxation of an elastic precursor and the stages of failure. The investigation of authors [4] is devoted to the development of structural-phenomenological models that take into account the link of structural changes caused by defects [5] and the relaxation properties of metals.

In the paper [6] an relaxation model is proposed that reflects the influence of dislocation ensemble kinetics on plastic flow taking into account the dependence of stress relaxation times on the dislocation density parameter according to the activation kinetics. This model, which reflects the kinetics of defects nucleation and growth, allows the quantitative description of shock wave fronts propagation in metals. Wide-range constitutive equations of plastic flow (strain rates $10^{3}-$ $10^{8} \mathrm{~s}^{-1}$ ) are proposed in $[7,8]$ for the interpretation of phenomenological assumptions of the MTS-PTW model related to the metastability of the thermodynamic potential for solid with defects, the nature of threshold flow stresses in the moderate range of strain rates of $10^{4}-10^{5} \mathrm{~s}^{-1}$ and the links the with asymptotic (self-similar) behavior of the plastic wave fronts (the Barker-Swegle-Grady fourth power law).

A special part is devoted to the study of the mechanisms of plastic strain localization and the stages of failure as a special type of critical behavior of solid with mesoscopic defects - structural-scaling transitions accompanied by the formation of collective modes of mesoscopic defects. These collective modes have the nature of self-similar solutions for the evolutionary equation of damage kinetics (autosoliton modes in the case of the development of plastic instability and dissipative structures for damage localization) and play the role of "collective variables" that subordinate the spatialtemporal dynamics of the behavior of condensed matter under intense impacts. The formation of self-similar plastic wave fronts [2,3], instability and damage-failure transitions in metals and ceramics [9-15], kinetic regularities of spall fracture in metals and ceramics $[12,15,16]$ can be regarded as the most striking manifestations of such space-time dynamics. The listed effects are studied theoretically $[6,11,13,16-22]$ on the basis of developed continuum models reflecting the role of metastability, collective modes of defects on the relaxation properties of solid and damage-failure transition scenario. Numerical simulation results are compared with the data of original experiments [23-28], which are used to identify the parameters and the explanation of the self-similar patterns of solid responses on shock wave loading.

Present study includes an analysis of the models of elastoplastic materials under shock wave loading taking into account the link between the relaxation mechanisms and the stages of failure with the nonlinear kinetics of mesoscopic defects (microcracks, microshears); identification of model parameters based on the results of dynamic experiments; numerical simulation of plane shock wave loading; verification of the model based on the comparison with the results of original experiments on shock wave loading of samples (metals and ceramics); substantiation on the basis of the results of numerical simulations and experimental data of self-similar propagation of shock wave fronts, the stages of failure in metals and ceramics.

With reference to numerical simulation, the behavior of materials under dynamic and shock wave loading, the justification of the model in a wide range of load intensities are related to elastic-plastic transition under the formation of shock wave fronts and damage kinetics.

\section{RELAXATION TIMES}

$\mathrm{T}$ he phenomenology of solid with defects contains a description of the thermodynamic state of the system based on the introduction of parameters that characterize the behavior of the ensemble of defects and have the meaning of independent thermodynamic variables. Within this approach, irreversible deformation is divided into plastic (dissipative) strain and structural strain, which allows us to separate the physical mechanisms underlying each of them. In 
this case, both the processes of defect accumulation and plasticity contribute to stress relaxation. Stress relaxation is determined by the nucleation and growth of defects in the shock front and should depend on the effective viscosity, which is the main stress relaxation factor that needs to be identified from experimental data. In the present paper, these parameters depend in general on many thermodynamic variables and can be represented by following the approach of the kinetic theory [29] as

$$
\tau_{i}=\tau_{0 i} \exp \left(\frac{U_{0}-\gamma \Omega}{\theta}\right)
$$

where $\theta$ is the temperature factor, $U_{0}$ is the characteristic value of the energy of the interatomic bond rupture, $\tau_{0 i}$ is the characteristic relaxation times, $\Omega$ is the generalized thermodynamic force, which in the Zhurkov model coincides with the applied stresses [12, 14].

In the works of the authors $[6,18]$, the dependence of the relaxation times on the accumulated strain in the form

$$
\tau=\tau_{0} \exp \left(\frac{\sigma_{t}+H \varepsilon-b \varepsilon^{2}}{\sigma}\right)
$$

where $\varepsilon, \sigma$ - the intensity of strains and stresses, respectively, $\sigma_{t}$ - yield stress, $H, b-$ the hardening and depletion coefficients, respectively. The generalized thermodynamic force depends on both stress and strain. It is necessary to assume the influence on the relaxation times of only the structural (inelastic) component, for example, in polymers practically coincides with the total strain [18]. The relaxation times for inelastic strain can depend on the accumulated structural strain due to defects [6]. In this case, the energy factor in Eqn. (1) is the thermodynamic force, which tends to return the system to the equilibrium state. Therefore, the relaxation times will be less than the system is further from the equilibrium state. Then Eqn. (1) for the model of solid with mesoscopic defects can be represented by

$$
\tau_{i}=\tau_{0 i} \exp \left(\frac{U_{0}-\gamma J\left(\Sigma-\rho \frac{\partial \Phi}{\partial E^{p}}\right)}{\theta}\right)
$$

where $J(\cdot)$ is the intensity of the tensor argument, $\boldsymbol{\Sigma}$ is the stress tensor, $\Phi$ is the specific free energy, $\partial \Phi / \partial \boldsymbol{p}$ is the nonlinear component of the thermodynamic force, which depends only on the inelastic strain $\boldsymbol{p}$ due to the defects. Comparison of Eqs. (2) and (3) shows that the energy contribution to the relaxation processes is divided into the effect of applied stresses and the structural component, expressed in nonlinear dependence on the characteristic deformations, as a polynomial for the model presented in $[1,9,18]$ or as nonlinear dependence of the component of the thermodynamic force due to defects [6].

\section{STRUCTURAL-STATISTICAL MODEL OF ELASTOVISCOPLASTIC DEFORMATION AND FAILURE OF SOLID WITH MESOSCOPIC DEFECTS}

$\mathrm{T}$ he approach developed by the authors is based on the description of the collective behavior of an ensemble of mesoscopic defects using the effective field theory for multiscale interaction between defects [5, 30]. Based on the geometric features of defects as a local changing in the symmetry of the distortion field (deformation) the nucleation and the growth of defects are associated with the contribution of induced by defects strain that is introduced as an additional structural variable. Taking into account the interaction of defects within the framework of the developed statistical-thermodynamic model it was possible to propose a continual description of solid with defects to formulate a system of constitutive equations linking the mechanisms of structural relaxation caused by the nucleation and growth of defects with the kinetics of elastic-plastic transitions and the stages of failure. An important point in the describing of mechanical properties of elastoplastic material is the established relationship between the formation of collective modes of defects ensembles related to the kinetics of plastic strain and damage localization. Variables for characterizing of defects 
(microcracks and microshears) are introduced like dislocation density tensors and are described by symmetric strain tensors in the case of microcracks $s_{i k}=s v_{i} v_{k}$ and microshears $s_{i k}=1 / 2 s\left(v_{i} l_{k}+l_{i} v_{k}\right)$. Here $\boldsymbol{v}$ is the unit vector to the base plane of the microcrack or slip plane of the microscopic shear, $\boldsymbol{l}$ is the unit vector in the direction of the shift, $s$ is the volume of the microcrack or the microshear intensity. The averaging of the microscopic tensor $s_{i k}$ gives a macroscopic tensor of microcracks (microshears) density (defect density tensor)

$$
p_{i k}=n\left\langle s_{i k}\right\rangle
$$

which coincides with macroscopic strain induced by defects, where $n$ is the concentration of defects.

Statistical theory for mesoscopic defects has established various qualitative reactions of the material represented in the nonlinear form of out-of-equilibrium free energy of solid with defects $F$ depending on defect density tensor $p_{i k}$ and the structural-scaling parameter $\delta_{c}$ characterizing the current susceptibility of the material to the nucleation and growth of defects. This parameter represents the ratio of the characteristic structural scales $\delta \sim\left(R / r_{0}\right)^{3}$, where $\mathrm{R}$ is the distance between the defects, $r_{0}$ is the average size of the defect nuclei. It was shown that in the intervals $\delta<\delta_{c} \approx 1, \delta_{c}<\delta<\delta_{*} \approx 1.3$ the responses of the material are characteristic for quasi-brittle, ductile and fine grain state, respectively $[5,30]$.
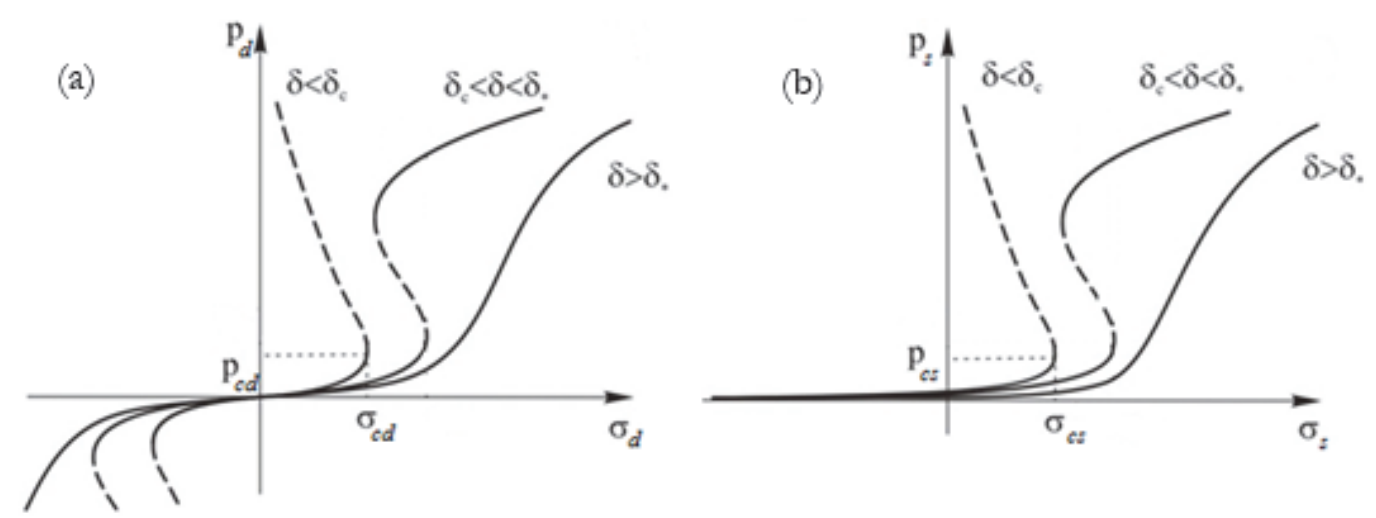

Figure 1: Characteristic material reactions on the growth of defects: (a) - for microshears, (b) - for microcracks

The curves in Fig. 1 demonstrate the solutions of the equation $\partial F / \partial p=0$ for simple shear $\left(\sigma=\sigma_{d}, p=p_{d}\right)$ and uni-axial strain $\left(\sigma=\sigma_{s}, p=p_{s}\right)$ states. Metastability for stresses $\sigma<\sigma_{c i}\left(\sigma<\sigma_{c d}\right.$ for microshears, $\sigma<\sigma_{c s}$ for microcracks) is a consequence of the effect of the ordering (orientation transition) in the ensemble of defects.

The value of stress $\sigma=\sigma_{c s}$ for $\delta<\delta_{c}$ defines the dynamic elastic limit (HEL) for quasi-brittle materials. The stress in the metastable area $\delta_{c}<\delta<\delta_{*}$ corresponds to the range of the elastic limit for materials with a plastic response (Fig. 1) [5].

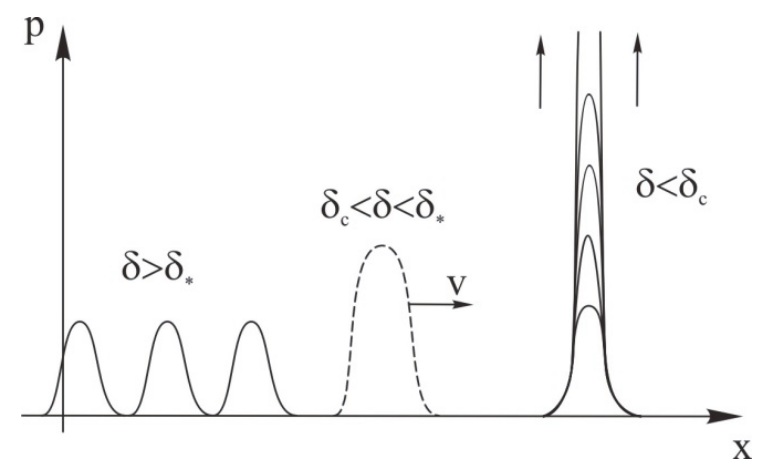

Figure 2: Self-similar solutions of the kinetic equation for strain induced by defects: periodic spatial structures $\left(\delta>\delta_{*}\right)$, autosoliton waves $\left(\delta_{c}<\delta<\delta_{*}\right)$ and localized dissipative structures $\left(\delta<\delta_{c}\right)$ 
For the interval of the structural scaling parameter $\delta_{c}<\delta<\delta_{*}$, the metastability regions in the continuum with defects appear. At a certain value of stress, an orientation transition occurs in the defects ensemble that leads to a sharp jump of plastic strain. This transition has a dynamic image (self-similar solution [5]) of an autosoliton wave (Fig. 2).

The transition through the critical value $\delta_{c}$ is accompanied by the formation of localized blow-up modes (Fig. 2), characterized by rapid kinetics of nucleation and growth of defects. The blow-up regime is the final stage of damage accumulation before the transition to fracture.

\section{NUMERICAL SIMULATION OF VANADIUM BEHAVIOR UNDER SHOCK-WAVE LOADING}

he mathematical modeling of dynamic loading of materials need the correct formulation of a system of equations that reflects physical and mechanical aspects for carrying out a computational experiment. It is of fundamental reason to establish the connection between the mechanisms of structural relaxation caused by the collective behavior of defects, the kinetics of the elastic-plastic transition and failure to describe the qualitative changes in the deformation reactions of materials with increasing load intensity (strain rates).

In this section, a mathematical statement of the collision of two plates is formulated using the constitutive equations taking into account the results of the statistical thermodynamic description of the collective behavior of defects (microshears, microcracks) and transition to elastoviscoplastic response and failure of metals (vanadium) due to defect induced metastability of thermodynamic responses of solid.

Mechanisms of plasticity and damage localization caused by the collective behavior of ensembles of defects are described taking into account the kinetics of two structural variables: the defect density tensor (defect-induced strain) and the structural-scaling parameter which determines the current susceptibility" of solid to the nucleation and growth of defects. Mechanisms of plastic deformation are associated with the kinetics of these two variables (microshears and microcracks density tensor) in the presence of metastability of thermodynamic potential providing a different scenario of defectinduced (structural) relaxation of stress in material.

The kinematic relation for solid with defects is given for the strain rates in the frame of small strains

$$
\dot{\varepsilon}=\dot{\varepsilon}^{e}+\dot{\mathbf{p}}+\dot{\boldsymbol{\varepsilon}}^{p}
$$

where $\varepsilon^{e}$ is the elastic strain, $\mathbf{p}$ is the strain induced by defects and $\varepsilon^{p}$ is the viscoplastic strain. Here and below, the tensor quantities are indicated in bold.

The thermodynamic description of the behavior of solid with defects is based on the phenomenological representation of the free energy using independent thermodynamic variables $\varepsilon^{e}, \mathbf{p}$ and $\delta$. Nonlinearity of thermodynamic potential in the Helmholtz form reflects the collective behavior of defects [5]. Taking into account the introduced thermodynamic variables and following the second law of thermodynamics the dissipative function of an elastoviscoplastic material with defects can be represented in the form

$$
T \dot{S}=\sigma \cdot \dot{\boldsymbol{\varepsilon}}^{p}+\left(-\frac{\partial F}{\partial \mathbf{p}}\right) \cdot \dot{\mathbf{p}}+\left(-\frac{\partial F}{\partial \delta}\right) \dot{\delta} \geq 0
$$

where $F$ is the free energy, $\delta$ is the parameter of structural scaling, $S$ is the entropy, $T$ is the temperature, $\sigma$ is the total stress.

Following the Onsager's principle the thermodynamic forces can be represented as linear combinations of thermodynamic fluxes

$$
\begin{aligned}
& \boldsymbol{\sigma}=\mathbf{A}_{1} \cdot \dot{\boldsymbol{\varepsilon}}^{p}-\mathbf{A}_{2} \cdot \dot{\mathbf{p}}, \\
& -\frac{\partial F}{\partial \mathbf{p}}=\mathbf{A}_{3} \cdot \dot{\mathbf{p}}-\mathbf{A}_{2} \cdot \dot{\boldsymbol{\varepsilon}}^{p}, \\
& -\frac{\partial F}{\partial \delta}=\mathrm{A}_{4} \dot{\delta},
\end{aligned}
$$


Here $\mathbf{A}_{1}, \mathbf{A}_{2}, \mathbf{A}_{3}$ are positive definite fourth-rank tensors, $A_{4}$ is a positive kinetic coefficient, which in the general case can depend on all the thermodynamic parameters.

Stress and strain tensors are represented as the sum of the bulk (index $s$ ) and deviator (index $d$ ) parts

$$
\begin{aligned}
& \boldsymbol{\sigma}=\boldsymbol{\sigma}_{s}+\boldsymbol{\sigma}_{d} \\
& \mathbf{p}=\mathbf{p}_{s}+\mathbf{p}_{d}
\end{aligned}
$$

Hooke's law in high-speed form was used

$$
\dot{\boldsymbol{\sigma}}=\lambda I_{1}\left(\dot{\boldsymbol{\varepsilon}}^{e}\right) \mathbf{I}+2 G \dot{\boldsymbol{\varepsilon}}^{e}
$$

where $\lambda$ is the Lame's first parameter, $G$ is the Lame's second parameter (the shear modulus), $I_{1}\left(\dot{\boldsymbol{\varepsilon}}^{e}\right)$ is the first invariant of the elastic strain rate tensor and $\mathbf{I}$ is the unit tensor of the second rank.

The equation of balance for mass is taken in the form

$$
\dot{\rho}=-\rho \nabla \cdot \mathbf{v}
$$

where $\rho$ is the density, $\mathbf{v}$ is the particle velocity.

The equation for momentum conservation is given as

$$
\rho \ddot{u}=\nabla \cdot \sigma
$$

where $\rho$ is the density, $\boldsymbol{u}$ is the displacement vector.

As a result, the system of constitutive Eqs. (7), (10) together with the balance Eqs. (11),(12) for an elastoviscoplastic material with defects complete the formulation of the mathematical modeling problem [25]. The system of differential Eqs. (7) - (12) describes the relationship of the relaxation mechanisms with the kinetics of the microshear and microcracks defects, with the laws governing the evolution of elastoviscoplastic flow and damage localization for the plate impact statement.

The developed model has eleven scalar parameters that require the realization of identification procedures. The approach for identification of material parameters was developed in [31] and based on the minimizing of the difference between the numerical simulation and the experimental data for quasi-static and dynamic tests. To provide the correctness of the optimization procedure (according to Hadamard) the solution was realized using a series of initial conditions. Mathematical statement for optimization procedure is presented below

$$
\begin{aligned}
& J=J_{1} J_{2} \ldots J_{n} \rightarrow \text { min, } \\
& J_{n}=\sum_{k=1}^{K}\left(\Sigma_{k}\left(\dot{\varepsilon}_{n}, \Gamma_{\sigma}, \Gamma_{p d}, \Gamma_{\delta d}, \sigma_{c d}, p_{c d}, \delta_{d 0}\right)-\Sigma_{k}^{*}\right)^{2}, \\
& \frac{1}{\varepsilon_{0}} \frac{\partial \varepsilon}{\partial \tau}=\frac{\partial \Sigma_{d}}{\partial \tau}+\frac{\partial \eta_{d}}{\partial \tau}+\frac{\partial \varepsilon^{p}}{\partial \tau}, \\
& \frac{\partial \eta_{d}}{\partial \tau}=\Gamma_{p d}\left(-\frac{\partial \Psi_{d}}{\partial p_{d}}\right) \\
& \frac{\partial \varepsilon^{p}}{\partial \tau}=\Gamma_{\sigma} \Sigma_{d}, \\
& \frac{\partial \delta_{d}}{\partial \tau}=\Gamma_{\delta d}\left(-\frac{\partial \Psi_{d}}{\partial \delta_{d}}\right) .
\end{aligned}
$$


The estimation of the model parameters was carried out by the stress-strain diagrams over a strain rate range of quasistatic and dynamic tests. A modified search method was used for solving the optimization problem. The initial vector of parameter values and the initial step were chosen to calculate the optimizing function at each iteration step. An indicator that has a value $\{-1,0,1\}$ allows the determination of the step value. If the point corresponds to the value of the indicator equal zero, then the step decreases, otherwise the current point becomes the minimum. The procedure stops when the step becomes less than the specified accuracy. This method was previously used to determine model parameters for various materials [31]. Fig. 3 shows a comparison for the stress-strain diagrams in the experiment [32] and numerical calculations obtained as a result of identification of model parameters for vanadium at different strain rates.

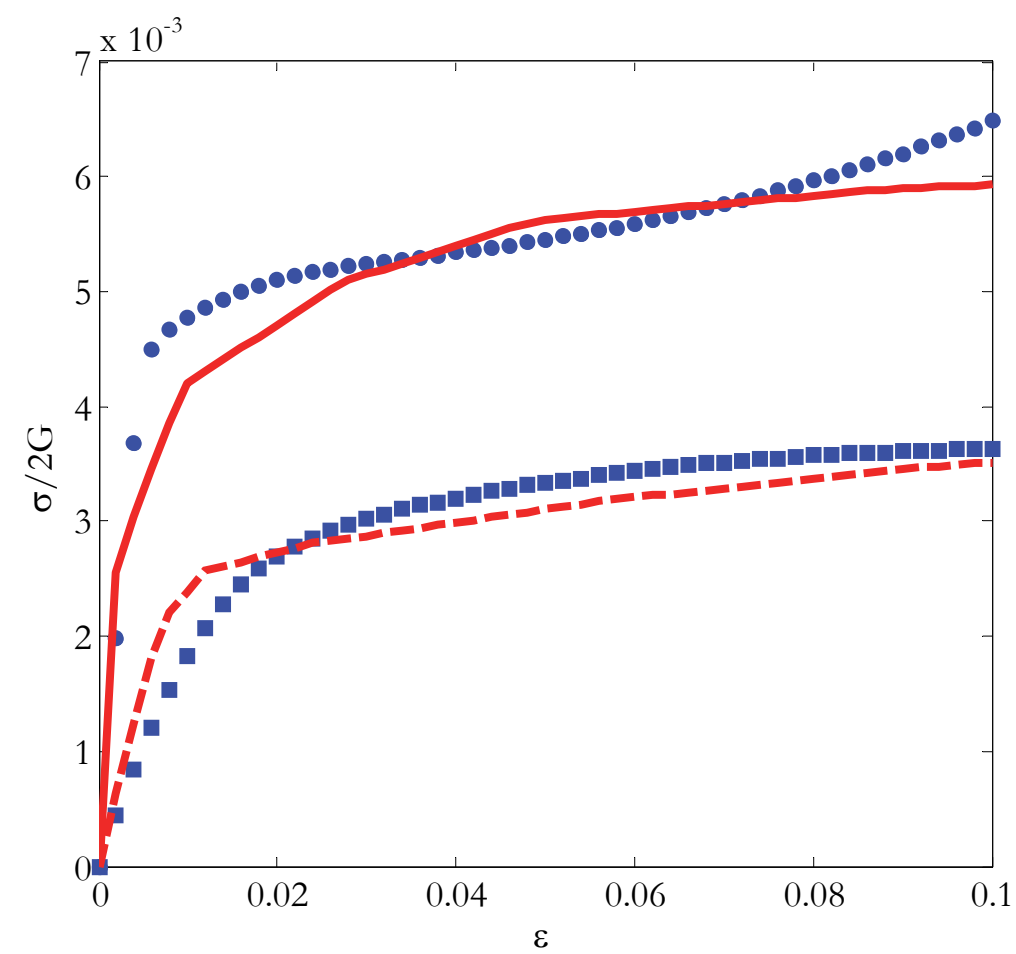

Figure 3: Experimental stress-strain diagrams for vanadium at different strain rates (dotted line $-10^{-1} \mathrm{~s}^{-1}$, dashed line $\left.-10^{3} \mathrm{~s}^{-1}\right)$ and corresponded numerical simulation $\left(\square-10^{-1} \mathrm{~s}^{-1}, \circ-10^{3} \mathrm{~s}^{-1}\right)$

It should be noted that the developed model makes it possible to describe stress-strain diagrams for wide-range strain rates $\left(10^{-1}-10^{3} \mathrm{~s}^{-1}\right)$ for the same values of model parameters. The procedure for verification of the model for vanadium was carried out from the shock-wave experiment in the conditions of a plate impact. Fig. 4 presents the results of numerical simulation and experiment [33].

Fig. 4 shows the particle velocity profiles for vanadium sample at different values of the stress amplitude that revealed the correspondence of numerical and experimental tests [33]. The profiles reflect the splitting of the shock wave front into an elastic precursor and the plastic front. The unloading of shock-compressed material behind the shock wave front leads also to the separation of the profile into an elastic wave and a wave of plastic unloading. In this zone, the numerical results do not agree well with the experimental profile, that can be linked with difficulty in recording the unloading wave front in the experiment.

The mathematical statement allows the modeling of the behavior of metals under shock wave loading in the presence of damage accumulation to predict the critical state of shocked material (damage localization as spall failure precursor). The spall failure is linked with the final stage of damage kinetics corresponding to the initiation of blow-up localized damage structures [5]. This stage occurs when the value of the bulk component $p_{s}$ of the defect-induced strain reaches the critical value $p_{c s}$ and appropriate distribution over the spatial scale according to blow-up (self-similar) collective modes.

The problem of spall failure for the plate impact test was studied numerically for vanadium. The simulation showed a significant dependence of the spall strength on the strain rate and typically (Fig. 5) the increase in spall strength for increasing strain rates [25]. 


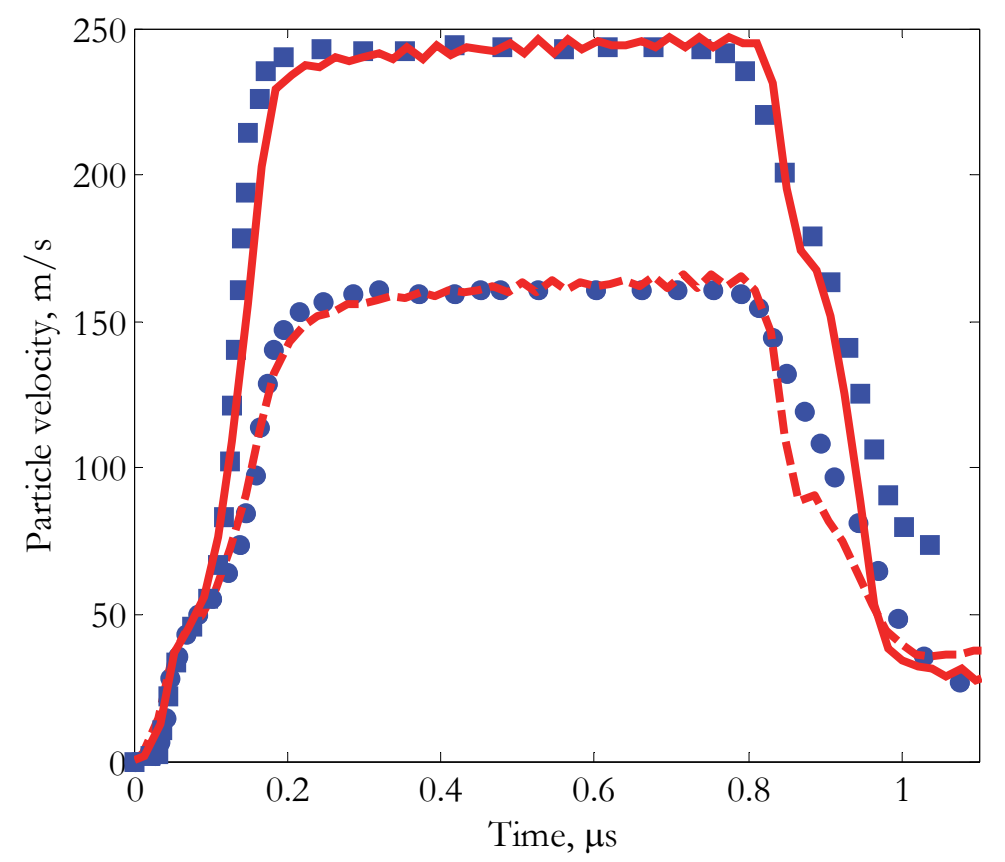

Figure 4: Particle velocity profiles for vanadium sample. The solid line is stress amplitude value about $9.7 \mathrm{GPa}$ (numerical calculation), the dashed line - 6.4 GPa (numerical calculation), o - 9.7 GPa (experiment), $\square-6.4 \mathrm{GPa}$ (experiment) [33]

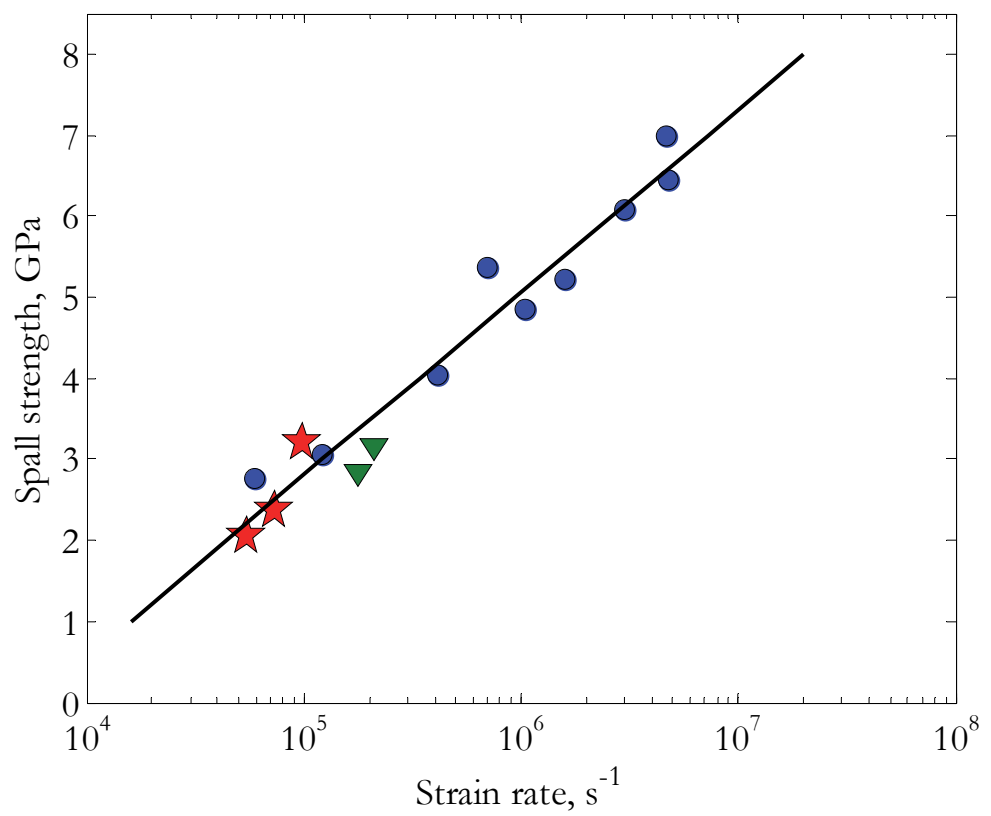

Figure 5: Dependence of spall strength versus strain rate: $\bullet$ - experiment [25], $\star$ - model calculation, $\boldsymbol{\nabla}$ - experiment [24]

The results of numerical simulation for the spall fracture are in accordance with the experiment and have established an increasing of spall stress with the increase of strain rate. That is the consequence of the pronounced damage localization with the avalanche kinetics of defect growth and weak sensitivity of the blow-up damage kinetics to the current value of stress amplitude.

During the numerical simulation, the velocity profiles were reconstructed at different parts of the sample in order to study the connection between the structural relaxation laws due to nonlinear defect kinetics, elastic-plastic transitions and the formation of viscoplastic wave fronts. Fig. 6 shows the results of numerical simulation and experimental dependencies of the elastic precursor amplitude for different thickness of the sample. 


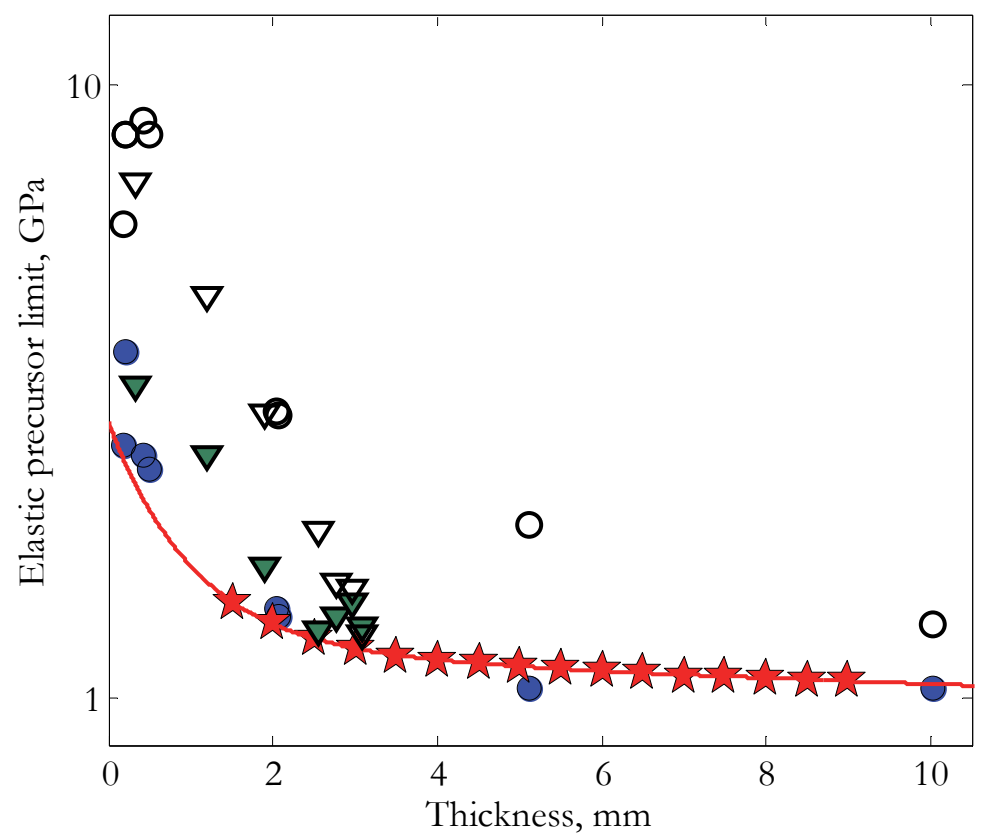

Figure 6: Dependence of the dynamic elastic limit on the thickness of the vanadium sample. Marker $\bullet$ is the minimum value (experiment) [25], ○ - maximum value (experiment) [25], $\boldsymbol{\nabla}$ - minimum value (experiment [24]), $\nabla$ - maximum value (experiment [ $25]), \star-$ numerical calculation, solid line - approximation.

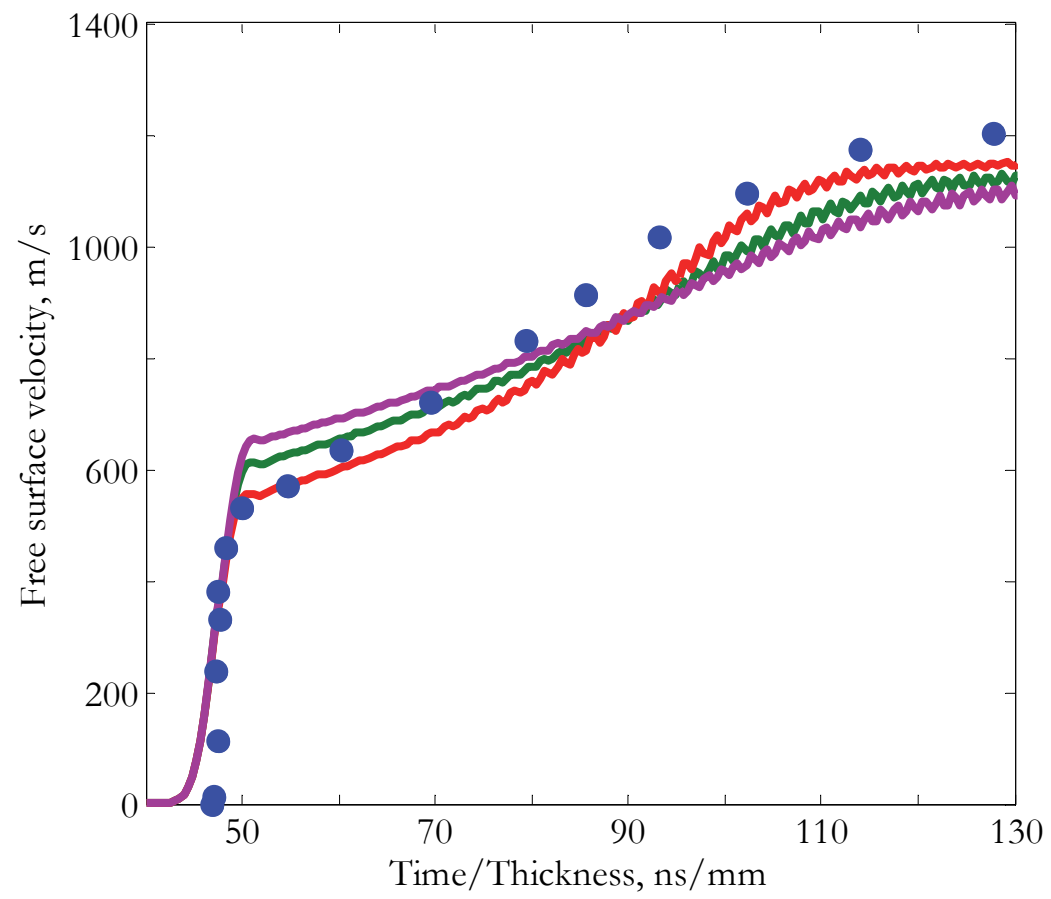

Figure 7: Free surface velocity profiles for silicon carbide samples in normalized time coordinates: 0 - experiment [15], lines numerical simulation for different target thicknesses $(8.32 \mathrm{~mm}, 3.81 \mathrm{~mm}, 2.15 \mathrm{~mm})$ [22].

The model describes the elastic precursor decay under the path of the elastoplastic wave in the sample (Fig. 6) that is in the agreement with the experimental data. The results of the simulation were approximated (solid line) by an exponential dependence in the form $\sigma_{H E L}=3.349 \exp (-0.962 h)+1.707 \exp (-0.036 h)$, where $h$ is the thickness of the sample.

Numerical simulation shows that the relaxation mechanisms of the elastic precursor can be associated with the effects of energy absorption due to the defects as a new "phase" of the material. This is based on the nonlinear kinetics of 
nucleation and growth of defects with pronounced transitions at metastability area in terms of the structural variable (the defect density tensor and structural scaling parameter).

\section{NUMERICAL SIMULATION OF SILICON CARBIDE BEHAVIOR UNDER SHOCK-WAVE LOADING}

7 he wide applications of ceramics have been found due to the high dynamic elastic limit and the ability to absorb the energy at high load intensities. Ceramics show a low level of plastic (viscoplastic) deformation and the elasticplastic transition in ceramic are associated with the "geometric" contribution of defects to the total strain. Fig. 7 shows a comparison of simulation and experimental results for silicon carbide under shock wave loading [15, 22].

The presented velocity profiles demonstrate the splitting of the shock wave into an elastic precursor and a plastic front. The value of plastic strain for ceramics is much smaller than that observed for metals. Weak sensitivity of the plastic front to the pulse intensity reflects the low contribution of the viscoplastic component, which is associated with low mobility of defects in ceramics. It was noted in [15] that some spall experiments in ceramics show weakly expressed spall pulse. This feature can be associated with a very short time of initiation and size of damage localization zone leading to small changes in the mechanical impedance during the formation of the spall surface.

The self-similarity of wave fronts in $\mathrm{SiC}$ ceramics was established in [15] and it was shown that the value of the dynamic elastic limit does not depend on time. In this connection, velocity profiles were constructed in time-normalized coordinates for different thicknesses of the samples (Fig. 7). This result demonstrates the self-similarity of shock wave fronts in ceramics, which is due to self-similar patterns of damage kinetics in the metastability area of the nonequilibrium thermodynamic potential for solid with defects.

\section{SELF-SIMILARITY OF SHOCK WAVE FRONTS IN METALS AND CERAMICS}

$\mathrm{F}$ or a wide class of materials (metals [1-3] and nonmetals [2]), the fourth power-law universality of plastic wave fronts is established (Fig. 8 and Fig. 9)

$$
\dot{\varepsilon}^{*}=A \cdot P_{0}^{\beta}
$$

where $P_{0}$ is the stress amplitude, $\beta$ is the exponent equal to four in the strain rate range $\dot{\varepsilon}^{*} \sim 10^{5}-10^{7} \mathrm{~s}^{-1}$ [3]. An explanation for the power universality of plastic wave fronts was proposed in [4], associated with the subjection of relaxation mechanisms to multiscale collective autosolitary modes of defects.

It is established that the fourth power-law is violated for vanadium [3,33]. Comparison of the results of the numerical simulation with experimental data is shown in Fig. 10. With an increase of strain rate at the wave front an asymptotic approximation to the fourth power-law is possible (in the graphs it is indicated by a solid line).

\section{CONCLUSIONS}

7 he mechanisms of structural relaxation are associated with collective behavior of mesoscopic defects related to the metastability of nonequilibrium potential of solid with defects and the generation of collective modes responsible for plastic strain and damage localization. These modes represent the self-similar solutions of the evolution equation for the structural variable (defect-induced strain), which determines the relaxation properties of material and scenario of elastic-plastic transition. Spatial-temporal dynamics of these modes (autosolitary and blow-up dissipative structures) can be initiated at appropriate load condition (shock wave rise) and could provide the anomalous relaxation ability of nonlinear system "solid with defects" in the conditions of the specific type of criticality - structural-scaling transition.

The metastability "decomposition" leads to the splitting of the wave front on the elastic precursor, the transient area of elastic precursor decay and the steady plastic front due to continuous (structural-scaling) transition between similar types of metastability depending on the shock wave amplitude. Autosolitary wave modes which subject the relaxation properties of shocked materials represent the "universality class" providing the four power universality at the steady shock wave front. The splitting of the wave front starts at the onset of critical behavior in the presence of metastability caused by the 
initial material structure. As the consequence, the elastic decay dynamics could be sensitive to the qualitative different relaxation kinetics: exponential kinetics with characteristic times that are close to the acoustic times and intermittent "slow" relaxation leading to the power law plastic wave fronts.

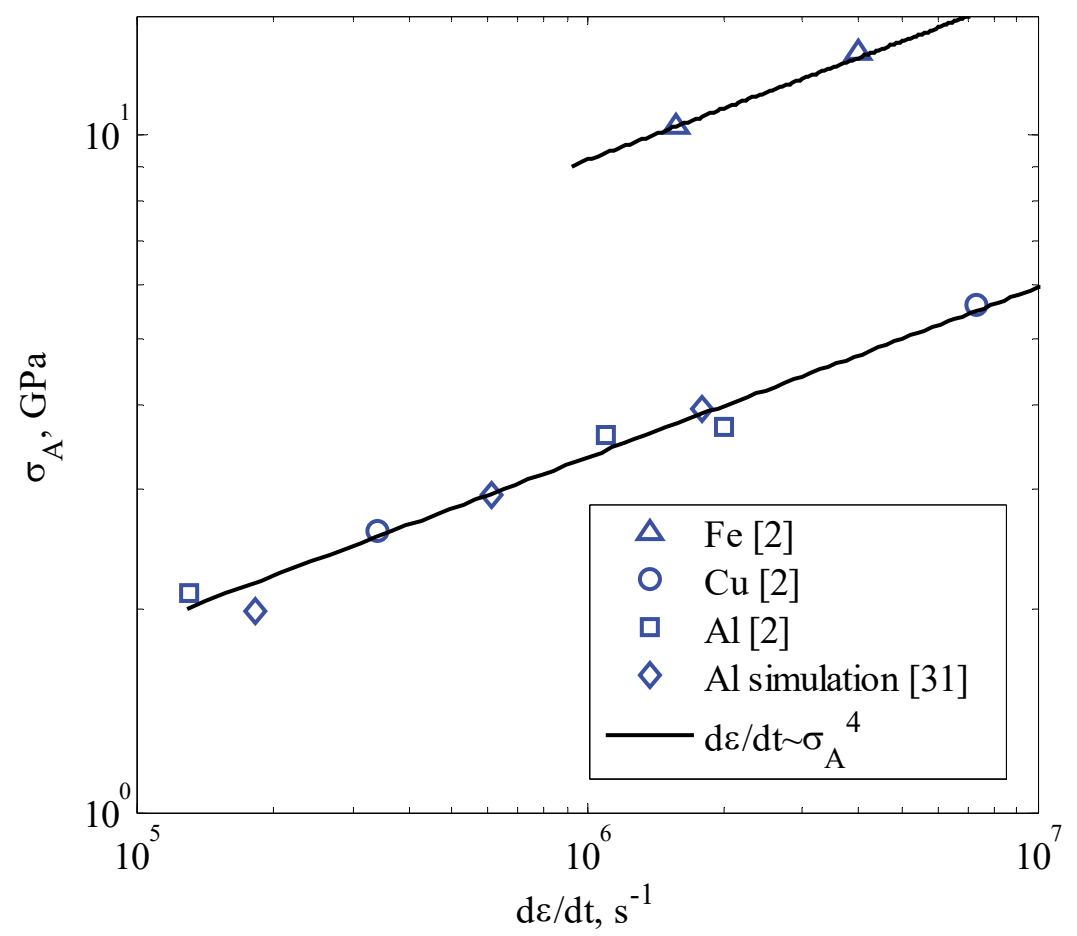

Figure 8: Dependences of the stress amplitude versus the strain rate at the shock front for metals

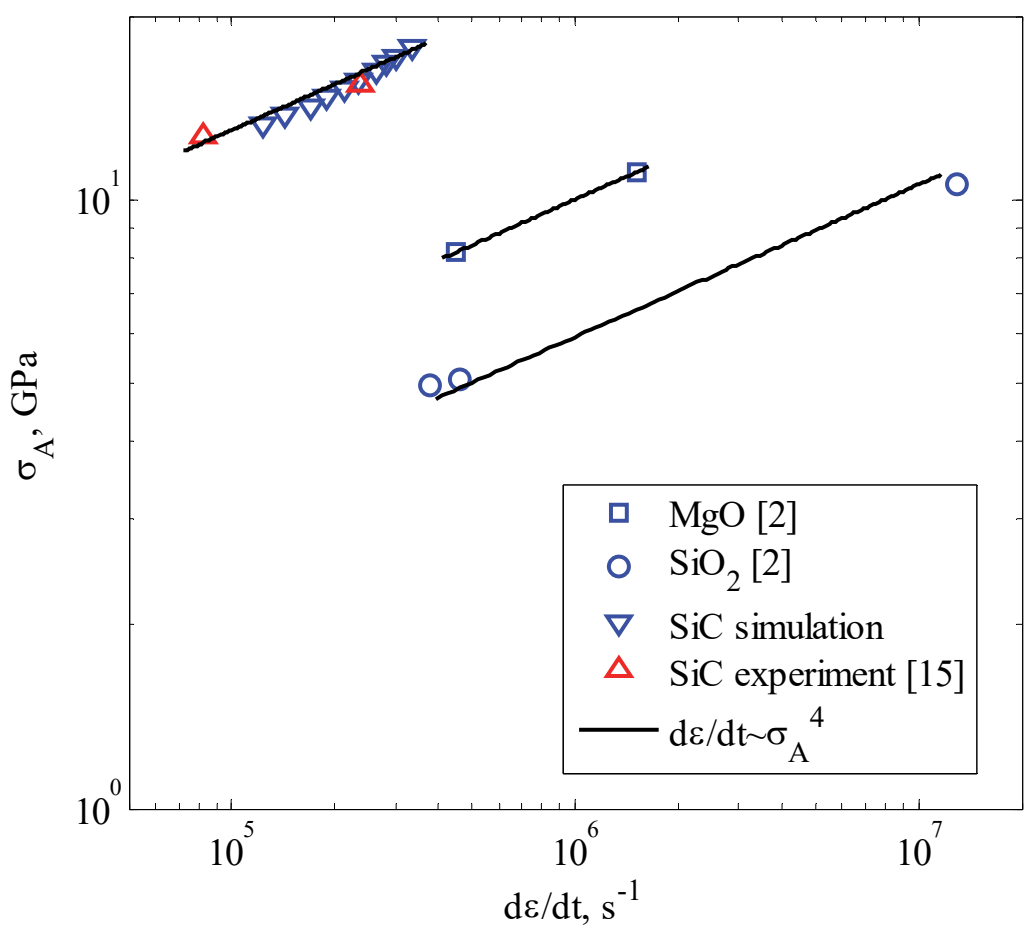

Figure 9: Dependences of the amplitude of stresses versus the strain rate at the front of the shock wave for nonmetals 


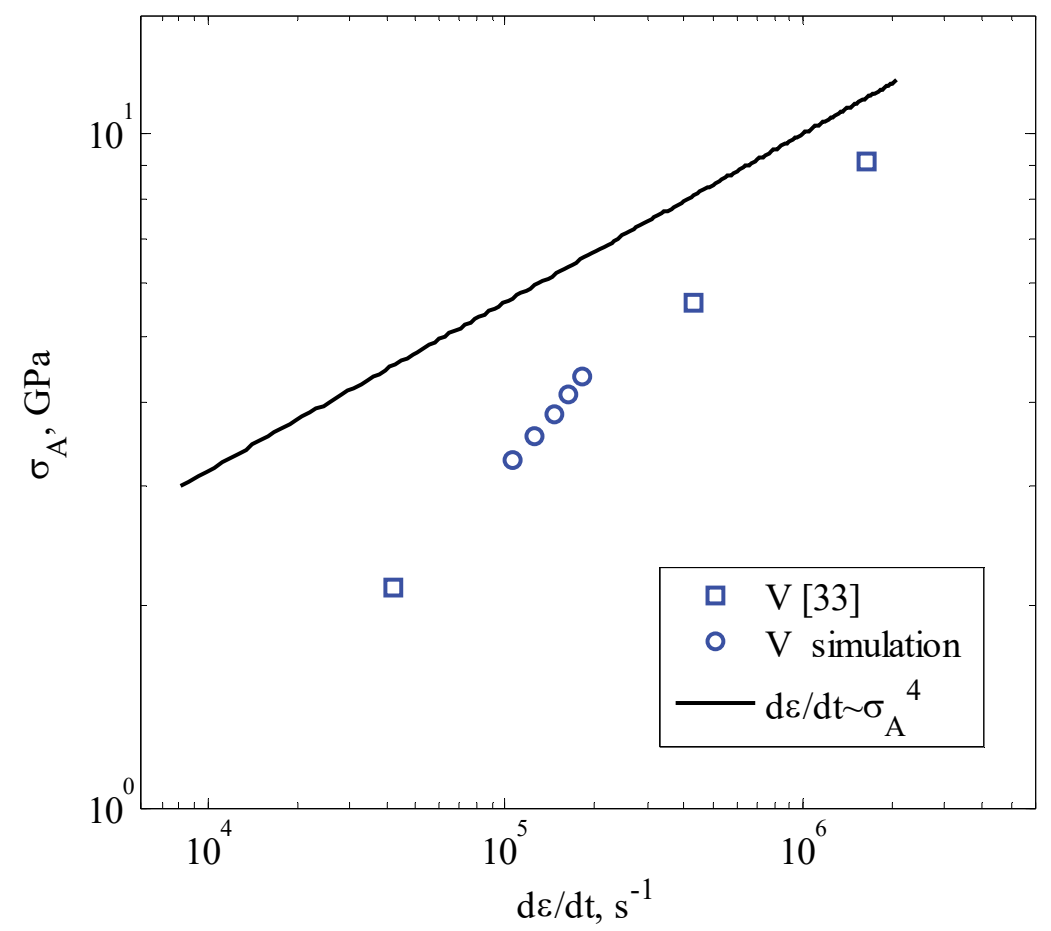

Figure 10: Dependences of the stress amplitude versus the strain rate at the front of the shock wave for vanadium

An explanation of the influence of the spatial-temporal dynamics of defects leading to the plastic strain instability (selfsimilar plastic wave fronts, adiabatic plastic shear) was proposed in $[4,34,35]$, on the kinetics of spall failure in metals and ceramics in $[22,25]$. The listed effects were studied theoretically on the basis of developed continuum models reflecting the role of metastability in the formation of collective modes and their relation to the relaxation properties of solid with defects. The results of the simulation are supported by original experiments that are used to identify the parameters and the results of the simulation to explain the self-similar features of deformation and fracture for metals and ceramics under shock wave loading.

\section{ACKNOWLEDGMENTS}

his work was supported by the Russian Foundation for Basic Research (project No. 17-01-00867).

\section{REFERENCES}

[1] Barker, L.M. (1968). Behavior of dense media under high dynamic pressures, Gordon and Breach, New York.

[2] Swegle, J.W., Grady, D.E. (1985). Shock viscosity and the prediction of shock wave rise times, J. Appl. Phys., 58(2), pp. 692-701. DOI: 10.1063/1.336184.

[3] Grady, D.E. (2010). Structured shock waves and the fourth-power law, J Appl Phys, 107, pp. 013506. DOI: $10.1063 / 1.3269720$.

[4] Bayandin, Yu.V., Leontiev, V.A., Mikhailov, E.V., Naimark, D.O., Savinykh, A.S., Skakun, S.N. (2004). Experimental study of wave fronts and structural scaling in copper upon shock-wave loading, Phys. Mesomech., 7(1-2), pp. 97-101.

[5] Naimark, O.B. (2003). Collective properties of defect ensembles and some nonlinear problems of plasticity and fracture, Phys. Mesomech., 6(4), pp. 39-63. 
[6] Merzhievskii, L.A., Paletsky, A.V. (2001). Calculations for diagrams of dynamic deformation of metals and alloys, Phys Mesomech., 4(3), pp. 85-96.

[7] Preston, D., Tonks D. (2003). Model of plastic deformation for extreme loading conditions, J App Phys., 93(1), pp. 211-220. DOI: $10.1063 / 1.1524706$

[8] Follansbee PS, Kocks UF. (1988) A constitutive description of the deformation of copper based on the use of the mechanical threshold stress as an internal state variables, Acta Metall., 36(1), pp. 81-93.

DOI: 10.1016/0001-6160(88)90030-2 .

[9] Makarov, P.V. (2004). On the hierarchical nature of deformation and fracture of solids and media, Phys. Mesomech., 7(3-4), pp. 21-29.

[10] Kanel, G.I., Razorenov, S.V., Fortov, V.E. (2004). Shock-wave phenomena and the properties of condensed matter, Springer, New York.

[11] Bayandin, Yu.V., Naimark, O.B., Uvarov, S.V. (2010). Numerical simulation of mesodefect induced spall failure under shock wave loading of metals, Computational continuous media mechanics, 3(1), pp. 13-23 (in Russian). DOI: 10.7242/1999-6691/2010.3.1.2.

[12] Petrov, Y.V., Smirnov, V.I. (2010). Interrelation between the threshold characteristics of erosion and spall fracture, Tech. phys., 55(2), pp. 230-235. DOI: 10.1134/S1063784210020118.

[13] Saveleva, N.V., Bayandin, Yu.V., Naimark, O.B. (2012). Modeling of deformation and failure of metals in plate impact test, Computational continuum media mechanics, 5(3), pp. 300-307 (in Russian). DOI: 10.7242/1999-6691/2012.5.3.35.

[14] Petrov, Yu.V., Gruzdkov, A.A., Bratov, V.A. (2012) Structural-temporal theory of fracture as a multiscale process, Phys. Mesomech., 15(3-4), pp. 232-237. DOI: 10.1134/S1029959912020117.

[15] Savinykh, A.S., Razorenov, S.V., Kanel, G.I., Rumyantsev, V.I. (2013). Evolution of shock waves in sic ceramic, Tech. phys., 58(7), pp. 973-977. DOI: 10.1134/S1063784213070207.

[16] Bayandin, Yu.V., Saveleva, N.V., Savinykh, A.S., Naimark, O.B. (2013). Numerical simulation of shock wave loading of metals and ceramic, Physics of extreme states of matter, pp. 64-67.

[17] Merzhievskii, L.A., Tyagel'skii, A.V. (1994). Modeling of dynamic compression of porous iron, Combustion, Explosion and Shock Waves, 30(4), pp. 522-530. DOI: 10.1007/BF00790160.

[18] Makarov, P.V. (2008). Mathematical theory of evolution of loaded solids and media, Phys. Mesomech., 11(5-6), pp. 213-227. DOI: 10.1016/j.physme.2008.11.002.

[19] Malygin, G.A., Ogarkov, S.L., Andriyash, A.V. (2013). On the Power-Law Pressure Dependence of the Plastic Strain Rate of Crystals Under Intense Shock Wave Loading, Physics of the Solid State, 55(4), pp. 780-786. DOI: $10.1134 /$ S1063783413040197.

[20] Malygin, G.A., Ogarkov, S.L., Andriyash, A.V. (2013). Two-Wave Structure of Plastic Relaxation Waves in Crystals Under Intense Shock Loading, Physics of the Solid State, 55(11), pp. 2280-2288. DOI: 10.1134/S1063783413110152.

[21] Malygin, G.A., Ogarkov, S.L., Andriyash, A.V. (2014). Dislocation Structure of Plastic Relaxation Waves in Polycrystals and Alloys Under Intense Shock Wave Loading, Physics of the Solid State, 56(11), pp. 2239-2246. DOI: $10.1134 /$ S1063783414110183.

[22] Bayandin, Yu.V., Saveleva, N.V., Savinykh, A.S., Naimark, O.B. (2014). Numerical simulation of multiscale damagefailure transition and shock wave propagation in metals and ceramics, Journal of Physics: Conference Series, 500, p. 152001. DOI: $10.1088 / 1742-6596 / 500 / 15 / 152001$.

[23] Kanel, G.I., Savinykh, A.S., Garkushin, G.V., Razorenov, S.V. (2015). Dynamic strength of tin and lead melts, Journal of Experimental and Theoretical Physics Letters (JETP Letters), 102(8), pp. 548-551.

DOI: $10.1134 /$ S0021364015200059.

[24] Zaretsky, E.B., Kanel, G.I. (2014). Tantalum and vanadium response to shock-wave loading at normal and elevated temperatures. Non-monotonous decay of the elastic wave in vanadium, J. Appl. Phys., 115(24), pp. 243502. DOI: $10.1063 / 1.4885047$.

[25] Saveleva, N.V., Bayandin, Yu.V., Savinykh, A.S., Garkushin, G.V., Lyapunova, E.A., Razorenov, S.V., Naimark, O.B. (2015). Peculiarities of the elastic-plastic transition and failure in polycrystalline vanadium under shock-wave loading conditions, Tech. Phys. Lett., 41(6), pp. 579-582. DOI: 10.1134/S1063785015060292

[26] Kanel, G.I., Garkushin, G.V., Razorenov, S.V. (2016). Temperature-Rate Dependences of the Flow Stress and the Resistance to Fracture of a VT6 Titanium Alloy under Shock Loading at a Temperature of 20 and $600^{\circ} \mathrm{C}$, Tech. Phys., 61(8), pp. 1229-1236. DOI: 10.1134/S1063784216080132.

[27] Kanel, G.I., Zaretsky, E.B., Razorenov, S.V., Ashitkov, S.I., Fortov, V.E. (2017). Unusual plasticity and strength of metals at ultra-short load durations, Physics-Uspekhi, 60(5), pp. 490-508. DOI: 10.3367/UFNe.2016.12.038004. 
[28] Petrova, A.N., Brodova, I.G., Razorenov, S.V. (2017). Strength properties and structure of a submicrocrystalline AlMg-Mn alloy under shock compression, Physics of Metals and Metallography, 118(6), pp. 601-607. DOI: 10.1134/S0031918X17060072.

[29] Zhurkov, S.N. (1984). Kinetic concept of the strength of solids, Int J Fract., 26(4), pp. 295-307. DOI: $10.1007 / \mathrm{BF} 00962961$.

[30] Naimark, O.B., Bayandin, Y.V., Zocher, M.A. (2017). Collective Properties of Defects, Multiscale Plasticity, and Shock Induced Phenomena in Solids, Phys Mesomech., 20(1), pp. 10-30. DOI: 10.1134/S1029959917010027.

[31] Bayandin, Y.V., Naimark, O.B., Uvarov, S.V. (2008). Structural-scaling transitions under dynamic and shock wave loads in solids, Physics of extreme states of matter, pp. 122-124 (in Russian).

[32] Tonks, D.L. (1991). The DataShop: a database of weak-shock constitutive data, Los Alamos, New Mexico.

[33] Chhabildas, L.C., Hills, C.R. (1986). in Metallurgical applications of shock-wave and high-strain-rate phenomena, Marcel Dekker, Inc.

[34] Bilalov, D., Sokovikov, M., Bayandin, Yu., Chudinov, V., Oborin, V., Naimark, O. (2015). Numerical simulation and experimental investigation of strain localization in AlMg6 alloy under dynamic loading, AIP Conference Proceedings, 1683, pp. 020025. DOI: 10.1063/1.4932715.

[35] Bilalov, D., Sokovikov, M., Bayandin, Yu., Chudinov, V., Oborin, V., Naimark, O. (2016). Numerical simulation of plastic strain localization and failure mode transition in metals under dynamic loading, Procedia Structural Integrity, 2, pp. 1951-1958. DOI: 10.1016/j.prostr.2016.06.245. 